\title{
REVISIONES
}

\section{Ley de desarrollo profesional docente en Chile: de la precarización sistemática a los logros, avances y desafíos pendientes para la profesionalización}

\begin{abstract}
Law on Teacher Professional Development: From systematic precariousness to the achievements, the advances, and the remaining challenges for professionalization.
\end{abstract}

\section{Andrea Ruffinelli Vargas}

Universidad Alberto Hurtado. Correo electrónico: aruffine@uahurtado.cl

\section{RESUMEN}

Ensayo que analiza la ley de desarrollo profesional docente recientemente promulgada en Chile, a la luz de dos perspectivas profesionales: orgánica y tecnológica. El documento recorre el largo y complejo proceso que culmina en una ley que regula la formación y ejercicio docente en el país, desarrollando un ejercicio analítico respecto de la racionalidad de la historia de sistemática precarización de la profesión docente que antecede a esta ley y de los eventuales alcances de su impacto en la orientación de la profesionalización del profesorado, a partir de los logros, avances, silencios y desafíos pendientes de la nueva ley, en el contexto del escenario social, económico, político y educativo de la nación.

Palabras claves: Desarrollo profesional docente, formación de profesores, ejercicio profesional docente, profesionalización docente.

\section{ABSTRACT}

This essay analyzes the recent Law on Teacher Professional Development in Chile, considering two professional perspectives: organic and technological. The document covers the long and complex process that culminates in a law that regulates teacher training and practice all around the country, developing an analytical exercise about the rationality of the history of systematic precariousness of the teaching profession that precedes this law and the possible extent of its impact on the orientation of the professionalization of teachers, from the achievements, progress, silences and remaining challenges of the new law, in the context of social, economic, political and educational scenario of the nation.

Keywords: teacher professional development, teacher training, teaching practice, teacher professionalization 
La idea de contar con una carrera docente en Chile es de larga data, y surge de la necesidad de responder a las necesidades de un país que decide reconocer el valor de sus maestros y el carácter estratégico de dicha profesión, comprendiendo que esa valoración no puede ser solo discursiva, sino que debe basarse en políticas que la posicionen de ese modo.

El horizonte de esta idea era un profesor que debía ser formado con la más rigurosa calidad, que debía ejercer bajo unas condiciones laborales y de desarrollo profesional acordes a la envergadura de la tarea, y que debía poder retirarse de la profesión bajo condiciones también acordes al rol desempeñado, todo esto, retroalimentado en una sociedad que valore profundamente la profesión. Esta aproximación surge de diversos estudios que muestran que los sistemas con mejores resultados educativos ubican la profesión docente entre las más valoradas, mejor remuneradas y más exigentes, pues la calidad de los profesores afecta directamente el aprendizaje y desarrollo integral de sus estudiantes, posibilitando el desarrollo armónico de la sociedad (Ingersoll, 2011; OCDE, 2004).

Esta idea también parece inspirarse en una concepción del docente como profesional -asumida como universal y única-, en términos de autonomía en la capacidad de tomar decisiones pedagógicas que deriven en un desarrollo integral de niños, niñas y jóvenes, a partir de unos saberes especializados, experiencia, reflexión y trabajo colaborativo.

Sin embargo, la concepción profesional del docente se plantea desde diversos enfoques en la literatura y cristaliza también de manera diversa en cada sistema educativo, al alero del contexto sociopolítico y económico en que se desarrolle.

\section{LA COMPRENSIÓN DEL ROL DOCENTE: PROFESIONALES TÉCNICOS/ BURÓCRATAS Y PROFESIONALES CLÍNICOS/ GESTORES SOCIALES}

Tanto en Chile como en otros países latinoamericanos la comprensión de la docencia como profesión aparece recién en las últimas décadas. Antes primaba una tradición identitaria magisterial funcionaria y técnica, que transitó desde el 'apostolado' -propio de la escuela gestionada por las iglesias- hacia una comprensión del docente como funcionario público -cuando el Estado irrumpe en educación. Luego, con la masificación educativa, se instala la concepción de docente como técnico, y solo en las últimas décadas se ha iniciado un camino hacia una comprensión 'profesional' (Núñez, 2004).

En este escenario, la definición de los sentidos y contenidos de la profesionalización es un objeto tensionado por los modelos sociales, educativos y económicos. Algunos autores han planteado modelos identitarios docentes dicotómicos. El modelo del 'management' (Dubet, Bergounioux \& Duru Bellat, 1999), o 'tecnológico' para Lang (2006), ligado a los principios de la burocracia: eficiencia, estandarización de objetivos, procedimientos y mediciones con resultados esperados predefinidos, que entiende al profesor como un tecnócrata, con un rol de didacta, siendo clave el componente científico técnico del oficio.

Como contrapunto, el 'modelo republicano' (Dubet et al., 1999), u 'orgánico' para Lang (2006), vinculado a lógicas basadas en consideraciones culturales, ético/morales y políticas, se sustenta en la confianza en la auto-regulación docente derivada de la autonomía profesional y de la responsabilidad del colectivo docente. Entiende al profesor como un profesional 'clínico', capaz de diagnosticar, definir estrategias de diversa índole y fines (no sólo instrumentales) y generar resultados de diversa naturaleza. Su rol es social y político, promotor de las causas de los derechos humanos universales. 
Desde esta perspectiva, y en consonancia con el modelo económico y societal imperante, veremos cómo en Chile se ha venido optando por el primer modelo.

\section{EL ESCENARIO EDUCATIVO CHILENO}

La opción por una concepción profesional docente desde la perspectiva del 'management' (gerencialismo) surge como correlato de decisiones políticas más sistémicas y profundas. La masificación del sistema escolar en la sociedad del conocimiento puso a las naciones en la encrucijada de optar por reformas educativas fundamentalmente de dos tipos: a) basadas en la equidad y el desarrollo de capacidades, y b) sustentadas en la rendición de cuentas e incentivos (Darling-Hammond, 2012). Las primeras, configuran sistemas educativos intelectualmente desafiantes, basados en un currículo reflexivo y en la promoción de habilidades complejas y colaborativas, avaladas por un alto nivel de calificación de sus docentes y sistemas de rendición de cuentas orientados a la evaluación permanente, con fines de retroalimentación y mejora de capacidades.

El otro grupo de reformas se vincula a la gobernanza escolar que discursivamente se refiere a los docentes como personas libres y capaces de construir su carrera, promoviendo la autonomía y autorregulación profesional, pero condicionándolos mediante estrategias de mercado (Feldfeber, 2007). Incorpora exámenes nacionales apoyados en motivaciones extrínsecas e invierte escasamente en capacidades docentes y en las escuelas, haciendo esfuerzos por individualizar y desprofesionalizar la docencia, pagando a sus docentes por mérito y atrayendo a personas sin preparación docente a la enseñanza, reduciendo la inversión en formación de profesores y desvinculándola de la academia, lo que genera cuerpos docentes con precarias competencias profesionales, que son estimulados a competir con sus pares. Someten a sus estudiantes y profesores a mediciones, con el fin de premiar/ castigar docentes y escuelas, desarrollándose un trabajo escolar orientado a responder tales pruebas, con el consiguiente estrechamiento del currículum y desvalorización de sus docentes.

Chile es uno de los países que ha orientado su reforma educativa en este último sentido (Darling-Hammond, 2012), y si bien es cierto que el campo de acción docente tiene una especificidad propia, dentro de la que debe actuar el profesor, sustentado en su preparación y juicio experto (Abbott, 1988), dicho campo es vulnerable a los cambios en estructuras fundamentales como el currículum, los modos de administrar el sistema educativo, y al nivel de prescripción externa sobre cómo ejercer la docencia (Tenti Fanfani, 2006, Day \& Gu, 2010).

\subsection{LA PARADOJA HISTÓRICA DE UN SISTEMA QUE PLANTEA DESAFÍOS PROFESIONALES A SUS DOCENTES Y EN LA PRÁCTICA LOS DESPROFESIONALIZA}

El discurso oficial contenido en instrumentos normativos de la práctica docente, tales como los Estándares orientadores para egresados de carreras pedagógicas (CPEIP, MINEDUC, 2011, 2012) y el Marco para la Buena Enseñanza (CPEIP, MINEDUC, 2008) releva una visión del profesor que se ajusta al primer grupo de reformas antes descritas, concibiéndolo en una categoría profesional. Sin embargo, este lenguaje discursivo cohabita -y lo viene haciendo desde hace unos cuarenta años- con políticas que apuntan en sentido contrario, y que paradójicamente son justificadas como medios para alcanzar la profesionalización de los docentes y el mejoramiento integral de los aprendizajes de los estudiantes. 
Estudios Pedagógicos XLII, N 4: 261-279, 2016

LEY DE DESARROLLO PROFESIONAL DOCENTE EN CHILE: DE LA PRECARIZACIÓN SISTEMÁTICA A LOS LOGROS, AVANCES Y DESAFÍOS PENDIENTES PARA LA PROFESIONALIZACIÓN

Los Estándares para egresados de carreras pedagógicas señalan que los docentes deben ser profesionales capacitados para el trabajo colaborativo, autónomo, flexible, innovador, dispuestos al cambio y proactivos, además de contar con una sólida formación en valores y comportamiento ético, manejo de comunicación oral y escrita en lengua materna y segunda lengua, habilidades en uso TIC, nivel cultural propio de mundo globalizado, así como espíritu de superación para ocuparse de su desarrollo personal y profesional permanente (CPEIP, MINEDUC, 2012).

En tanto, el Marco para la Buena Enseñanza describe al profesor como un profesional capaz de preparar adecuadamente la enseñanza, crear un ambiente propicio para el aprendizaje, enseñar a sus estudiantes de modo que aprendan, y asumir sus responsabilidades profesionales, reflexionando consciente y sistemáticamente sobre su práctica, y reformulándola a partir de dicha reflexión (CPEIP, MINEDUC, 2008).

Adicionalmente, este desempeño profesional es constantemente tensionado por un contexto socioeconómico, político y cultural que demanda a sus profesores no solo rápidas y certeras adecuaciones ante los cambios vertiginosos de la sociedad, de las políticas y del currículum que deben enseñar, sino también proactividad para el ejercicio en condiciones difíciles, con remuneraciones inferiores a profesiones comparables, con muy escaso tiempo para preparar y evaluar la enseñanza, enfrentando exigencias de manejo cabal de la diversidad, de la vulnerabilidad y desmotivación de sus estudiantes y familias, de las progresivas exigencias evaluativas ministeriales, y de las demandas sociales que exigen estar siempre actualizado, demandas que se incrementan continuamente, de manera que parece inversamente proporcional a las condiciones ofrecidas para alcanzar las capacidades necesarias y a la valoración social de la profesión, haciendo más difícil lo que ya lo es por naturaleza (Ávalos, 2013).

Desde esta perspectiva, el rol docente trasciende con mucho la enseñanza exitosa de un currículum explícito y a responder apropiadamente un test estandarizado, debiendo el profesor hacerse cargo de problemas estructurales de la sociedad, pese a los cuales se le demanda lograr aprendizajes integrales de calidad con equidad, cargando con la responsabilidad de desempeñar una profesión identificada como clave para el desarrollo del país, en un situación que difiere sustantivamente del contexto educativo anterior a la escolarización plena.

Sin embargo, las expectativas y exigencias de la más alta sofisticación profesional surgen en un escenario que venía avanzando por décadas en la implementación de políticas gerencialistas conscientemente articuladas, que implican nociones de profesionalización ligadas a incentivos individuales por resultados, tensionando la identidad docente histórica más vinculada a la ética del servicio público. Esta situación ha derivado en un sentimiento de desprofesionalización y desvalorización docente (Bellei, 2001), tanto en dictadura como en democracia, que han incluido:

a) En dictadura: cierre de las escuelas normales que, hasta 1974, se ocuparon de la formación de profesores; redefinición del rol del Estado en educación, asumiendo que el fin de la educación básica era capacitar para ser buenos trabajadores, ciudadanos y patriotas, y autorizando a los establecimientos a focalizarse en alfabetización y cálculo básico, lo que redunda en el constreñimiento de la formación de profesores y del currículum escolar, incluyendo la eliminación de contenidos que promovieran el pensamiento crítico en todos los niveles del sistema educativo. 
Desde 1981, eliminación del carácter universitario a la formación docente (Arévalo; Gysling \& Reyes, 2013), que se recupera sólo a partir del año 2015; y, simultáneamente, entrega de la formación de profesores a los principios de un mercado fuertemente desregulado, que deriva en una precarización de la calidad de la formación impartida (Cox; Meckes \& Bascopé, 2010).

En 1988, se levanta un pilar fundamental del gerencialismo educativo: la prueba nacional SIMCE, estandarizada, de lápiz y papel y selección múltiple, en algunas disciplinas consideradas fundamentales, que rinden censal y anualmente escolares de diferentes niveles educativos, presentándose estos resultados como indicadores de calidad de las escuelas e identificando a los establecimientos educativos en la comunicación de sus resultados, bajo la forma de rankings -hasta la medición del año 2012- y responsabilizando a las escuelas por los resultados, pese a que se asocian consistentemente al origen socioeconómico de los estudiantes y no a los efectos de su escolarización.

Esta información se hace pública, esperando que estimule la competencia entre escuelas para lograr más matrícula, y derivan en estrechamiento curricular y en una formación y ejercicio docente acotado a prescripciones que garanticen buen rendimiento en estas pruebas, desvalorización del rol docente y deterioro del clima escolar (Alexander, 2010, 2011; Hargreaves \& Fullan, 2012).

b) En democracia: prontamente se establece un Estatuto Docente (1991), que ofrece un trato especial al profesorado, tras décadas de precarización de la profesión regida por las condiciones de las actividades privadas. El Estatuto regula temas relativos a condiciones de trabajo, como remuneraciones, jornadas de trabajo, condiciones difíciles, bonos de perfeccionamiento y experiencia, y garantiza estabilidad laboral, pero no se hace cargo de los efectos de la promoción de la regulación vía mercado de la formación docente. Es así como la esencia gerencialista del modelo societal y educativo no solo no fue modificada en democracia, sino que fue sofisticada (Osborne \& Gaebler, 1993). Tuvo lugar un crecimiento explosivo y desregulado de las carreras de pedagogía, alcanzando a más de 1.500 programas diferentes de pedagogía y más de 120.000 estudiantes, con un incremento de la matrícula superior al 200\% en diez años (Cox, Meckes \& Bascopé, 2010); en heterogéneas condiciones de exigencias de ingreso, egreso y oportunidades formativas, sobre un escenario básico de precariedad, entre cuyos estudiantes uno de cada tres no rindió la prueba de selección universitaria (pues no existían exigencias de requisitos de ingreso a carreras pedagógicas), y entre los que lo hicieron más del 70\% logró menos de 500 puntos -el promedio del instrumento-, además de ofrecerse unas perspectivas laborales difícilmente atractivas, al ser una de las profesiones peor remuneradas.

El acceso democrático a carreras de pedagogía, como argumento de reivindicación social -amparado por la desregulación estatal-, confunde el derecho a la educación con el derecho a ser profesor. Cuatro décadas de discurso aspiracional y meritocrático individual persuadieron a un país respecto a que todos deben acceder a la educación superior para 'ser alguien', y que una de las alternativas más accesibles y de bajo costo, incluyendo financiamiento estatal, es la de estudiar pedagogía.

Cualquier universidad -y hasta el año 2014, cualquier Instituto Profesional sin ofrecer el grado de Licenciatura- podía impartir una carrera pedagógica, en las condiciones que estimara convenientes, pese a la obligatoriedad de acreditación de estos programas formativos -que rige desde el año 2007-, pues resulta exigible solo para efectos de 
acceso a financiamiento público. Es decir, una carrera pedagógica no acreditada podía operar sin problemas si sus estudiantes lo financiaban. Adicionalmente, los dictámenes de acreditación no ofrecían garantía de calidad (Meckes y Domínguez, 2011), eran disímiles y omitían dimensiones claves para evaluar una formación de calidad (por ejemplo, resultados formativos), focalizándose en condiciones de operación (Domínguez, Bascopé, Lorca y Pozo, 2012). A esto se suman evidentes conflictos de interés en el proceso, puesto que las instituciones son clientes de las agencias acreditadoras (Cox et al., 2010).

A nivel escolar, la reforma curricular del año 1996 fija el centro en Lenguaje y Matemática, reforzando el carácter alfabetizador de la educación básica, y el inicio del doble estándar el año 2009, con la Ley General de Educación que determina nuevos objetivos orientados a la formación integral como sujetos autónomos y reflexivos, elaborando unas Bases Curriculares en tal sentido (2012). Sin embargo, el sistema de evaluación vuelve a relevar como prioridad Lenguaje y Matemática (Arévalo, Gysling \& Reyes, 2013).

En 2003, se implementó la política docente, ícono del gerencialismo: la evaluación y los incentivos al desempeño docente, orientando explícitamente a los profesores hacia el modelo de profesionalismo propio de este enfoque, que es percibido por ellos como ajeno a su rol (Sisto, 2011). Políticas de profesionalización similares, sustentadas en el desempeño, se encuentran con frecuencia también en las reformas educativas de otros países latinoamericanos (Tenti, 2006) y en Europa (Gunter, 2008). La principal tensión docente frente a esta evaluación estriba en la reconceptualización que hace de su trabajo, privilegiando el desempeño individual por sobre el colectivo, estandarizando y prescribiendo, y con ello restringiendo, la posibilidad de vincularse a su práctica habitual, debiendo mostrar evidencias ajustadas a categorías prefijadas (Sisto, 2011).

En la misma línea del doble discurso, los estándares, la rendición de cuentas y el control, surge el año 2008 otro pilar del gerencialismo: la evaluación de egreso a la profesión docente, la prueba INICIA, de lápiz y papel y selección múltiple, midiendo conocimientos disciplinarios y pedagógicos de los estudiantes de pedagogía al terminar su proceso formativo, restringiendo en este acto el rol docente a estos dominios y formas de evidencia de sus saberes, y que desde su versión del año 2010 identifica a las instituciones y las rankea, sin considerar que por sobre todo y al igual que SIMCE, da cuenta de las diferencias socioeconómicas de origen de los egresados y mide de modo altamente sesgado la calidad formativa de las instituciones.

La evaluación de diferencias entre los programas de la oferta formativa debe ser asumida por el consumidor/cliente, haciendo uso de las asimétricas herramientas de discernimiento disponibles -como los resultados de la prueba INICIA-, dejando recaer toda la responsabilidad de la elección del programa sobre el postulante y su familia, enfrentando instituciones e individuos a las consecuencias de unos resultados que se asumen de su exclusiva responsabilidad, reservándose para el Estado el rol de informador.

Las entidades formadoras no están obligadas a alinearse a la prueba INICIA con la que se les mide. Sin embargo, lo hacen porque obtienen consecuencias positivas al hacerlo (prestigio ante la opinión pública, incremento de matrícula, etc.). Así es como el mecanismo de control opera y modifica la conducta de las instituciones y de los postulantes, legitimando un concepto de calidad vinculado y restringido a lo que las pruebas externas miden.

Como corolario, para la desprofesionalización, en el año 2009 se promulga el artículo 46, letra 'g' de la Ley General de Educación, que permite a titulados de cualquier carrera de ocho semestres de duración hacer clases en la enseñanza media o secundaria, reconociendo 
de este modo que no se requieren saberes profesionales específicos para el ejercicio de la docencia (Cox, Meckes \& Bascopé, 2010). Un nuevo instrumento de control que disemina la idea de un profesor sin estatus profesional, que difiere del discurso oficial que le advierte que será juzgado con los más altos estándares profesionales, y en la práctica implementa políticas que lo desvalorizan y lo miden a partir de un concepto restringido de la docencia.

En suma, un discurso oficial promotor de profesionales reflexivos y críticos, y medios de control en sentido inverso, es decir, un doble discurso. Paradojalmente, se fustiga a los profesores como responsables individuales de los resultados educativos, los propios y los de sus estudiantes -propio de enfoques gerencialistas-, omitiendo la responsabilidad de un sistema que desprofesionaliza y que mantiene expectativas y exigencias de desempeño profesional.

Desde la perspectiva de los resultados precarios que han alcanzado sistemáticamente en distintas mediciones nacionales e internacionales nuestros docentes y sus estudiantes en las escuelas, y particularmente la prueba INICIA, indicando que la mayoría de los docentes recién egresados no cuenta con los saberes mínimos necesarios para desempeñarse idóneamente, son resultados que no consideran lo que aparece como más relevante en la interpretación de estos resultados: la responsabilidad sobre la ausencia de itinerarios formativos que se hicieran cargo de la aceptación de postulantes con capacidades insuficientes para el desafío y sobre la ausencia de garantías de calidad en la formación.

En este escenario, es preciso también destacar que desde los años noventa se han impulsado políticas tendientes a mejorar la formación inicial de los docentes, y con ello a incentivar su profesionalización. Sin embargo, el carácter prescriptivo y acotado de estas políticas, cuyo alcance solía ser el de los programas tradicionales y de mayor prestigio, abandonaba a la suerte de la desregulación y la falta de apoyo a la mayoría de los programas y estudiantes de pedagogía.

En Chile -como en otros países-, se experimenta la tensión provocada por la consolidación de un paradigma educativo basado en estándares, competencia individual y pruebas externas de altas consecuencias en parcelas del conocimiento, con exigencias de rendimientos que se espera lograr en base a incentivos, asumiendo que basta con presionar para obtener mejores resultados, distorsionando el ideario de desarrollo integral y reemplazándolo por un escenario de desprofesionalización de la docencia, en oposición a una mirada de generación de competencias profesionales en entornos colaborativos, con escuelas que funcionan bien, y no profesores aislados que aseguran bien o menos bien unos resultados en unas pruebas externas reduccionistas de altas consecuencias.

Los países con buenos resultados han optado por una combinación de presiones e incentivos con apoyos a la generación de condiciones para la enseñanza y el aprendizaje, y el desarrollo de capacidades, con una rendición de cuentas orientada a la retroalimentación y mejora en lugar de la sanción punitiva. La apuesta central se da, entonces, por el desarrollo de capacidades y la colaboración, en oposición a los incentivos, rendición de cuentas, sanciones y competencia individual, capaz de alcanzar y retroalimentar una alta valoración social de esta profesión.

Según el estudio TALIS (2013), más de un tercio de los docentes chilenos estima que su profesión es valorada por la sociedad, ubicándose por sobre el promedio de los países participantes en ese estudio. Sin embargo, en países que muestran los mejores resultados educativos, esta valoración positiva supera con creces el 50\% de la población. En Chile, las encuestas CIDE 2008, 2010 y 2012, muestran una realidad similar, con alrededor de un $23 \%$ de los docentes que estima que la profesión docente es valorada socialmente. 
Estudios Pedagógicos XLII, N 4: 261-279, 2016

LEY DE DESARROLLO PROFESIONAL DOCENTE EN CHILE: DE LA PRECARIZACIÓN SISTEMÁTICA A LOS

LOGROS, AVANCES Y DESAFÍOS PENDIENTES PARA LA PROFESIONALIZACIÓN

\section{LA NUEVA LEY DE DESARROLLO PROFESIONAL DOCENTE: EL COMPLEJO PROCESO Y SU ROL EN LA PROFESIONALIZACIÓN DE LA DOCENCIA}

El acuerdo en la idea de legislar para regular la formación y ejercicio docente evidenció un consenso social inédito hacia una comprensión de que el mejoramiento de la educación implica que sus docentes sean capaces de promover aprendizajes significativos, bajo apropiadas condiciones de formación y desempeño, que no pueden ser reguladas por el mercado.

Sin embargo, la forma que debía asumir esta ley no era objeto de consenso. Esto, en un país altamente segmentado, que había experimentado más de cuatro décadas de degradación y devaluación social de la profesión docente, como consecuencia del desarrollo inversamente proporcional de las corrientes gerencialistas que entienden la educación de una nación -como todas sus demás dimensiones- como un bien de consumo transable en el mercado, cuyos actores deben regular individualmente su acción rigiéndose por los principios de ese mercado.

En este escenario, surgen sentimientos crecientes de desconfianza, pues es preciso desplegar todo tipo de artilugios para ganar esta competencia contra los otros y responder individualmente a unos estándares que se han fijado externamente. Es así como aparece la desconfianza del magisterio ante el proyecto original, que daba múltiples señales de inspiración gerencialista. Era difícil esperar que un sistema en que se había socializado a un país por décadas fuese capaz de generar modos genuinamente distintos de comprender la profesión docente.

Un extenso paro nacional de profesores puso en evidencia la historia, las fracturas, los miedos, las naturalizaciones, y generó conversaciones, propuestas y aprendizajes. Se reinventó el diálogo y pendiendo de un hilo, se reinventó la actual ley. El proyecto hizo un complejo recorrido en el parlamento por diez meses. A mitad de ese camino, el MINEDUC presentó más de 80 indicaciones a la propuesta original, a las que siguieron sumándose otras orientadas a promover mayor reconocimiento y confianza en los docentes, a valorar la naturaleza colaborativa del trabajo de los profesores, y a poner mayor énfasis en los apoyos al desarrollo docente que en las acciones punitivas y de control.

En este proceso, se observa la consecución de dos hitos como principios organizadores:

a) Una sola profesión docente, para la gran mayoría de los profesores chilenos, un sistema de desarrollo profesional para los docentes de todo el sistema subvencionado por el Estado. Queda pendiente la inclusión de los docentes del sistema particular pagado, para entender que existe una sola profesión docente para todos los profesores de Chile. Se trataba de una condición básica para comenzar a revertir la concepción mercantil del sistema educativo, pues cualquier regulación que no fuese aplicable al sistema particular subvencionado, implicaba un reconocimiento a la continuidad de un sistema que asume que el sector privado adoptará gradualmente estas regulaciones.

b) Una profesión docente construida con amplia participación ciudadana y docente, desde el MINEDUC y la sociedad civil. Un ejemplo de organización e incidencia en este sentido fue la iniciativa 'El Plan Maestro', que articuló las voces de más de 20 instituciones y de un foro base ciudadano con representantes de todos los concernidos en el tema educativo, que por más de dos años generaron discusión pública y entregaron al Ministro de Educación sus propuestas, que fueron consideradas en el texto final de la ley. 


\subsection{LOGROS, AVANCES Y DESAFÍOS PENDIENTES}

Durante la discusión del proyecto, se visualizaron al menos ocho dimensiones imprescindibles para avanzar hacia una profesión docente acorde con las necesidades del país. A continuación se plantea una discusión en torno al nivel de logro de cada una, a la luz de su incidencia en el tipo de profesionalización docente que la inspira:

a. Regulación de la oferta formativa y aseguramiento de un sistema formativo de alta calidad

b. Regulación del ingreso a carreras pedagógicas

c. Regulación de la calidad de los egresados de pedagogía

d. Regulación del ingreso a la profesión docente

e. Inducción o acompañamiento a la iniciación docente

f. Diseño de una carrera atractiva

g. Condiciones laborales específicas

h. Retiro consistente con la carrera

\subsubsection{Regulación de la oferta formativa y aseguramiento de un sistema formativo de alta} calidad: solo universidades y programas acreditados y evaluación

Que la formación inicial sea parte de la carrera docente constituye un logro en sí mismo. Las formas que adquiere esa incorporación son variadas. En Chile no estaba regulada la oferta de carreras pedagógicas, y con la nueva ley sigue sin regularse la oferta de programas, que es parte de la autonomía universitaria. Es decir, si eventualmente un programa cumple con los requisitos de acreditación, puede ofrecer la carrera, aunque no responda a las necesidades de formación de docentes del país.

Si bien la tendencia internacional muestra un rol activo del Estado para definir políticas de formación y determinar centralizadamente la cantidad de profesores que se requiere formar, en Chile no se dispone de estudios actualizados ni existen prescripciones respecto de cuántos profesores deben ser formados en cada especialidad. La oferta de carreras pedagógicas se incrementó en torno a un $200 \%$ entre los años 2000 y $2009^{1}$, y esta tendencia se detuvo a partir de 2013, probablemente a causa de las nuevas medidas de regulación e incentivos (estándares, prueba INICIA, beca vocación de profesor y sus condiciones).

Respecto de la acreditación de las carreras pedagógicas, como se señalara anteriormente, ya se establecía antes su obligatoriedad, aunque solo para efectos de recibir financiamiento público. Es así como el año 2012, en Chile, de las 901 carreras pedagógicas existentes, cerca del $24 \%$ no estaban acreditadas. De las acreditadas, una proporción marginal alcanzaba seis o siete años (máximo periodo de acreditación), mientras el grueso obtenía no más allá de cuatro ${ }^{2}$. Y ya se señalaba antes la ausencia de foco de esta acreditación en la calidad.

La nueva ley faculta solo a universidades y programas acreditados impartir carreras pedagógicas, y paralelamente aumentan los requisitos para lograr la acreditación que exclusivamente la Comisión Nacional de Acreditación (CNA) puede otorgar, en lugar de hacerlo las agencias acreditadoras, como ocurría hasta el año 2015. Los nuevos requisitos

http://www.divesup.cl/sies/?page_id=42

Ver en www.cnachile.cl 
de acreditación incluyen exigencias básicas de infraestructura y equipamiento, de cuerpos docentes idóneos, prácticas tempranas y progresivas, aplicación de evaluaciones diagnósticas y de medio término, dispositivos de seguimiento y apoyo al proceso formativo de los estudiantes, exigencia de coherencia entre itinerario formativo y perfil de egreso, y existencia de convenios con establecimientos educativos que aseguren un vínculo más sólido y estable entre ambas instituciones formadoras. Es decir, condiciones para la calidad.

De este modo, para esta dimensión del análisis, se observa una orientación de la ley en un sentido profesional de tipo más bien orgánico, al regular las condiciones de la calidad de la oferta de carreras pedagógicas. Sin embargo, mantiene el enfoque mercantil para efectos de la cantidad de la oferta.

Finalmente, en esta dimensión, encontramos dos ausentes: la ley no contempla la necesidad de contar con unos mínimos orientadores formativos comunes para el sistema que, considerando los sellos formativos de cada entidad formadora, garanticen un acuerdo nacional acerca de los elementos vertebradores de la formación; así como otro ausente sigue siendo la escuela y los profesores guías como agentes formadores fundamentales legitimados, en colaboración mutua con la universidad.

\subsubsection{Regulación del ingreso a carreras pedagógicas: un indiscutible avance, aunque dista de 'solo los mejores'}

En Chile tampoco existía regulación del ingreso a carreras pedagógicas. Hasta el año 2016, las instituciones formadoras determinaban si exigían o no rendición de la Prueba de Selección Universitaria (PSU) -único requisito, cuando se exigía alguno-, y eventualmente puntaje mínimo en ella.

Desde el año 1995, cerca de la mitad de los postulantes a carreras pedagógicas ingresó con menos de 550 puntos PSU (500 puntos equivale a poco más de 30\% respuestas correctas, y es el promedio de logro en esta prueba ${ }^{3}$ ), y el año 2014, el $29 \%$ de quienes estudian una carrera de educación lo hace sin exigencias de puntaje PSU. Los institutos profesionales -que hasta 2014 podían ofrecer carreras pedagógicas- no solo eran los que matriculaban menos estudiantes con PSU rendida, sino que también obtenían el promedio PSU más bajo, bordeando los 430 puntos. Por su parte, las universidades del Consejo de Rectores de las Universidades Chilenas (CRUCh) obtienen en promedio el puntaje PSU más alto, sobre 500 puntos. Las universidades privadas no CRUCh concentran la mayor parte de la matrícula, y puntajes promedio bajo 500 puntos.

La selectividad de los programas también varía según la región geográfica: por ejemplo, mientras el promedio PSU en las universidades del CRUCh de la Primera Región en Educación Parvularia no alcanza los 500 puntos, en la Región Metropolitana supera los 600 puntos (MINEDUC, 2012).

En la práctica, los postulantes de más bajos resultados o los que no han rendido PSU estudian carreras pedagógicas, y provienen de establecimientos educacionales de menor nivel socioeconómico respecto de estudiantes de otras carreras de duración similar: prefieren un sueldo estable por falta de redes para optar a mejores condiciones, en tanto, los estudiantes de grupos socioeconómicos altos no postulan a pedagogía por el bajo estatus social de la profesión docente (Munita, 2010). Asimismo, los estudiantes de instituciones

www.demre.cl 
de baja o nula selectividad provienen de establecimientos educativos de bajo prestigio y cuando egresan trabajan en establecimientos similares (Ruffinelli \& Guerrero, 2009; Ortúzar et al., 2009; Meckes \& Bascopé, 2012).

La ley innova al regular el ingreso a carreras de pedagogía, cuyos postulantes podrán ser seleccionados solo entre quienes estén dentro del 30\% de los mejores puntajes PSU de ese año, entre quienes se hayan ubicado en el $30 \%$ superior del rendimiento de su promoción en la educación secundaria, o bien entre quienes hayan cursado en las universidades un programa propedéutico con este fin. Ante requisitos de ingreso hasta hoy inexistentes esto representa un innegable avance, aunque no garantiza que los matriculados sean los 'mejores', como ocurre en otros países con mejores indicadores educativos que los chilenos, en que la rigurosa selección es un elemento fundamental del sistema. Y en esta línea argumentativa se omite deliberadamente la pregunta relativa a cuán 'buenos' son nuestros 'mejores', a partir de la precariedad de nuestros resultados educativos en el sistema escolar.

La actual PSU, sensible a los contenidos tratados en la enseñanza media, genera una situación de desventaja para los estudiantes cuyos establecimientos ofrecen oportunidades de aprendizaje de menor calidad, razón por la que resulta razonable incorporar mecanismos alternativos, como la selección de estudiantes que se ubiquen dentro del $10 \%$ de los mejores egresados de su promoción -criterio actualmente usado por los programas propedéuticos en curso-, independientemente de sus resultados PSU, sumados a programas de apoyo a la incorporación a la vida universitaria, y también utilizado por países que a nivel internacional muestran mejores logros educativos. También resulta razonable incorporar mecanismos complementarios, como entrevistas de admisión, para indagar acerca de las motivaciones y capacidades vinculadas a la pedagogía, aunque esto último no lo contempla la ley.

Se ha argumentado que el incremento sustantivo de estos requisitos podría ocasionar la ausencia de postulantes en regiones extremas. Por la misma razón, alternativas pudieron haber sido: a) generar condiciones excepcionales para dichas regiones, o b) regular de esta forma de manera transitoria, estableciendo una gradualidad en el incremento de exigencias hasta llegar a fórmulas que efectivamente aseguren una alta selectividad, junto a condiciones país que lo soporten.

Con todo, se trata de políticas inéditas, aportando al desarrollo profesional desde una perspectiva más orgánica, al regular y con ello revertir el enfoque mercantil en la selección de estudiantes.

\subsubsection{Regulación de la calidad de los egresados}

La ley incorpora dos evaluaciones censales a los estudiantes de pedagogía, una diagnóstica al inicio de la carrera (a cargo de las propias universidades) y otra un año antes de finalizar (en reemplazo de la actual INICIA). Esta medida reconoce la responsabilidad de las instituciones formadoras sobre los resultados de sus estudiantes, y obliga a implementar remediales cuando no se cumple lo esperado. Sin embargo, aparece como un avance perfectible. Una razonable alternativa sería desarrollar las evaluaciones de medio término y casi término con un carácter muestral en lugar del censal que se propone, con menos visos de rendición de cuentas y ranking, y más de evaluación formativa que retroalimente los procesos desarrollados por las entidades formadoras. También parece importante considerar que evaluaciones diagnósticas diversas, creadas por las propias universidades no serán 
de utilidad como línea base de comparación con resultados que se obtengan en la evaluación nacional de casi término.

Desde esta perspectiva, la ley avanza, al ubicar la responsabilidad en las instituciones. Sin embargo, el Estado aparece débil como regulador de la calidad, al optar por instrumentos de control, como acreditación y evaluaciones a los estudiantes, sin contrapuntos visibles en dispositivos de apoyo al fortalecimiento de los procesos formativos, como por ejemplo, los actuales convenios de desempeño, que bajo las condiciones vigentes, contribuyen a la reproducción de la estructura de un sistema formativo segmentado, pues son las mismas instituciones y programas las que sistemáticamente se adjudican dichos fondos, precisamente a causa de sus mejores credenciales. Es decir, un énfasis en la profesionalización desde una perspectiva más bien gerencialista.

\subsubsection{Regulación del ingreso a la profesión docente: ingreso automático}

La ley plantea como requisitos de ingreso a la carrera docente el título profesional y contrato de trabajo (vía concurso público, en el caso de establecimientos municipales). En el proyecto original, se supeditaba el ingreso a la carrera a un examen de certificación, que si bien apuntaba a asegurar la calidad docente en un contexto de heterogénea y precaria formación, profundizaba el problema haciendo responsables a los egresados de la desregulación de un sistema que ha permitido esto por décadas. Con la nueva ley, se ubica el foco en el aseguramiento de la calidad de la oferta formativa, en lugar de castigar a los egresados.

Con todo, en el frágil escenario actual, resulta razonable insistir en la gravitancia del proceso de aseguramiento de la calidad directamente sobre los programas formativos. La experiencia internacional indica que cuando el foco está puesto en el desarrollo de las capacidades docentes, a partir de una exigente selección de los candidatos, y en el fortalecimiento de la formación inicial y continua, la excelencia pedagógica es una consecuencia que no requiere estresantes dispositivos de control como las pruebas (Hargreaves, 2012).

Finalmente, es razonable que solo puedan ejercer la docencia profesores habilitados, reconociendo la indudable necesidad pero insuficiencia del manejo de una disciplina para ejercer la docencia. Sin embargo, este aspecto no fue considerado en la ley y se mantiene la vigencia de la disposición que permite ejercer la docencia en la enseñanza media a profesionales de cualquier ámbito con al menos ocho semestres de estudios superiores.

Es decir, se observa una orientación de la ley hacia la profesionalización orgánica, al fortalecer la responsabilidad institucional por sobre la individual y apostar a la formación especializada de calidad por sobre la certificación, aunque mantiene la asunción relativa a que no se requiere formación profesional especializada para ejercer la docencia, propio de los enfoques gerencialistas.

\subsubsection{Inducción a la iniciación docente: un derecho para todos los profesores principiantes}

La literatura ha sido amplia y profusa en el sustento teórico y empírico para justificar la necesidad de acompañar a los profesores en su iniciación a la docencia. Existe una gran diversidad de programas de inducción en el mundo. Suelen requerir de un mentor y de liberación de horas para mentor y principiante. Es etapa final de la formación en Francia y Alemania, posterior a la formación en Países Bajos y Reino Unido. En Chile, hasta la promulgación 
de esta ley no existía una política pública de inducción, solo escuelas que voluntariamente apoyan con dispositivos especiales la iniciación de sus docentes.

Según el informe TALIS 2013, en Chile, menos del 50\% de los directores de escuelas afirma que en sus establecimientos existen programas formales de inducción, y menos del $30 \%$ de los docentes afirma haber participado en alguno de ellos. El promedio para los países participantes es de $70 \%$ y 50\%, respectivamente, y solo para el $10 \%$ de los docentes sus directores señalan que los profesores disponen de un mentor en la escuela. Un estudio nacional muestra que el $60 \%$ de los egresados señala no haber recibido ningún apoyo de su institución formadora tras el egreso, y menos del $20 \%$ señala haber recibido algún apoyo de su escuela (Ruffinelli, 2013).

La literatura indica que los programas de inducción mejoran las prácticas docentes, los logros de aprendizaje escolar y la retención de profesores principiantes (OCDE, 2009; Ortúzar et al., 2011). Estas iniciativas requieren de la integración entre centros de práctica e instituciones formadoras, llevando a cabo en forma conjunta proyectos que se inician desde las experiencias prácticas de los profesores en formación, que culminan con un periodo de inducción no inferior a un año, acompañado por la institución formadora en colaboración con la escuela. Este logro constituye una decisión sin precedentes en nuestro país, que en el proceso legislativo transitó desde un acompañamiento voluntario sujeto a cupos hacia la mentoría como un derecho para todos los nuevos profesores.

Es altamente valorable la posibilidad que tendrán los establecimientos de desempeño alto y medio de generar sus propios planes de inducción. Sin embargo, la ley estipula que la inducción debe estar a cargo de mentores certificados. Dada la escasez de mentores certificados hay una alta probabilidad de ser acompañado por un docente que no es de la escuela, desconociendo por una parte la naturaleza contextual y situada de la mentoría, y por otra los hallazgos de investigación que relevan la importancia de un docente experimentado que acompañe, por sobre un docente certificado. Este solo hecho, independiente de la formación específica del mentor, hace la diferencia: es referido como el principal facilitador de la iniciación (Ruffinelli, 2014). En este escenario, las altas exigencias para ser mentor bien podrían ser progresivas, y optar por privilegiar que se garantice su participación intencionada en el proceso formativo.

Con todo, esta dimensión de la ley se ancla en un enfoque orgánico, en tanto recupera la mirada de construcción colaborativa, mediada, reflexiva y trascendente de la profesión.

\subsubsection{Carrera atractiva: Mejoramiento de remuneraciones iniciales, desarrollo profesional y derecho a formación permanente}

a) La progresión en la carrera

El sistema de reconocimiento del desarrollo profesional sintoniza con lógicas profesionalizantes de tipo orgánicas, en lugar de acercarse a lógicas gerencialistas del sistema de certificación propuesto originalmente. Tiene dos pilares: el reconocimiento al desarrollo, que implica mejoramiento inicial y progresivo de remuneraciones; y aseguramiento de formación continua que apoye el desarrollo profesional. Esta combinación incorpora una perspectiva orgánica al promover condiciones para el desarrollo profesional que si bien se retribuyen económicamente, no es lo único que ofrecer.

La carrera -de orientación horizontal- incentiva a mantenerse trabajando en el aula, se basa en resultados de evaluación, y se estructura en cinco tramos, tres de ellos obligatorios. 
Parecería razonable justificar los tramos voluntarios, por ejemplo, incorporándolos al desarrollo profesional fuera del aula; ya sea como requisitos para cargos de jefatura técnica, o de supervisión ministerial, profesionalizando así un rol que históricamente no ha tenido ese carácter. Sin embargo, la carrera no contempla distintas trayectorias, con distintas funciones a partir de los resultados alcanzados. También se ha señalado que esto podría desincentivar el interés por permanecer dentro del aula, o de la escuela, espíritu que ha animado la idea de esta carrera, que mantenga a los buenos profesores en las aulas.

Por su parte, la evaluación para el avance en la carrera contempla una prueba de conocimientos disciplinares y pedagógicos, en el nivel y en la especialidad, y un portafolio que evidencie el desempeño contextualizado, funciones docentes fuera del aula (trabajo colaborativo, rol en actividades del establecimiento) y el perfeccionamiento pertinente. Resulta valiosa la vinculación que hace la ley respecto de esta evaluación, a dos documentos antes señalados: el Marco para la Buena Enseñanza, y los estándares orientadores para la formación docente, y también valioso el reconocimiento al desarrollo profesional en la práctica y no solo a aquel ligado a procesos académicos formales certificados.

Respecto a la evaluación, la ley no se pronuncia respecto a la coexistencia o integración de dos sistemas: la evaluación docente (con fines retroalimentadores y formativos) y la evaluación para la progresión en la carrera. Con todo, la evaluación debería desplazar el foco desde la rendición de cuentas a la retroalimentación y mejoramiento profesional. Paralelamente a la discusión parlamentaria, se desarrollaban tres mesas de trabajo, una de ellas precisamente acerca del proceso evaluativo.

Hasta hoy, el agobio que se vincula a la evaluación docente guarda estrecha relación con su carácter no real. Las evidencias que se recogen no necesariamente forman parte de lo que habitualmente hace el profesor, y más bien se trata de portafolios creados para la ocasión, con la consiguiente energía adicional que eso implica. ¿Cómo avanzar a portafolios como evidencia de la práctica efectiva? Mejores procesos formativos tributarán a esto, generando autoconfianza, permitiendo que los profesores identifiquen y muestren lo mejor de sí. De todos modos, la ley contempla que buenos resultados evaluativos disminuyen las ocasiones en que es obligatoria la evaluación, reduciendo el agobio.

Uno de los elementos del portafolio, el perfeccionamiento, había sido desestimado en un inicio a partir de evidencia nacional relativa a que -cuando no está asociado a indicadores de calidad- no aporta información relevante para explicar los resultados de aprendizaje de los estudiantes (Bravo, Falck, González, Manzi y Peirano, 2008). Esta concesión a los maestros, que históricamente han financiado personalmente su perfeccionamiento, supone un gran desafío al fortalecimiento del CPEIP para el mejoramiento de la calidad y pertinencia del sistema de formación continua.

Respecto de la progresión, como efecto del ingreso automático a la carrera, el tiempo total de recorrido para llegar a las remuneraciones más altas disminuye, pues el nuevo profesor no debe esperar para certificarse, como proponía el proyecto original. Sin embargo, la exigencia de 4 años de experiencia para avanzar al tramo siguiente levanta la duda acerca de la legitimidad de impedir a un profesor con menos experiencia la promoción cuando evidencia contar con las capacidades.

Finalmente, resulta crucial avanzar hacia desplazar el foco desde desempeños y competencias individuales hacia prácticas de comunidades escolares, de escuelas que trabajan colaborativamente, hacia las metas que se proponen. Los incentivos deberían migrar del plano individual al colectivo. Insistir en los méritos individuales constituye un 
escollo de la ley para sintonizar con una visión de profesionalización más orgánica, aunque la incorporación de esta dimensión colaborativa en el portafolio, así como de la valoración del desarrollo profesional que se ha alcanzado en el ejercicio (trascendiendo a cursos certificados), incorporan valiosos elementos, aunque se midan de manera individual.

b) Las remuneraciones iniciales

La expectativa consideraba un incremento salarial sustantivo de base, que asegurara la atracción de los mejores, y que se homologara desde el principio con las remuneraciones de las profesiones más prestigiosas, de modo que estudiar pedagogía fuese una alternativa real frente a otras opciones a las que se puede acceder con el mismo puntaje PSU, y de modo que ayudara a reposicionar socialmente el rol del profesor. La ley avanza en este sentido, acercándolas a los $\$ 800.000$ en el inicio -un alza indiscutiblemente significativa-, aunque a costa de matizar los incrementos en los tramos siguientes y hacer con ello menos atractiva la carrera en su desarrollo.

Con todo, el ideario de la carrera debía ser cuidadoso con los incentivos, pues focalizar en ellos implica nuevamente una concepción neoliberal que asume que los profesores cuentan con las herramientas para desplegar un buen trabajo pedagógico y que simplemente requieren estímulos externos para hacerlo, sin considerar que podrían estar atrapados en la debilidad de sus saberes, situación que bajo ninguna circunstancia se supera con incentivos, sino con el fortalecimiento de la formación inicial y continua y del trabajo colaborativo. Desde esta perspectiva la ley resguarda ambas situaciones.

c) Formación continua como derecho: fortalecimiento del CPEIP y foco en la pertinencia Los esfuerzos de apoyo al desarrollo profesional docente se concentran en la formación continua, que se establece como un derecho cuya gestión queda a cargo del CPEIP, que para estos efectos se fortalece y crece en sedes y oferta, con énfasis en las necesidades reales de los docentes y de los establecimientos educativos, toda vez que las exigencias de perfeccionamiento para avanzar en la carrera obligan a su pertinencia. La ley establece prioridades: los profesores de establecimientos subvencionados y patrocinados por su sostenedor, los profesores principiantes, los de bajo desempeño y los que se desempeñan en establecimientos vulnerables y aislados, cambios que parecen razonables desde una orientación a las necesidades de profesionalización.

Resulta altamente valorable el reconocimiento al carácter situado y contextual de las necesidades de formación continua, al recaer en los establecimientos la definición de sus necesidades formativas y planes para satisfacerlas. Desde esta perspectiva, ciertamente se observa una orientación de la ley hacia la profesionalización orgánica, a partir de criterios de pertinencia y calidad, desde una perspectiva integral.

\subsubsection{Condiciones de trabajo: Incremento de las horas no lectivas y silencios}

Datos de la OCDE del año 2009 señalan que Chile es el tercer país que más horas destina a la docencia de aula, y el promedio de los países miembros no supera el 57\%. Asimismo, Chile está entre los tres países en que más profesores reportan nunca asistir a reuniones de equipo, cerca del 40\%, y más de la mitad nunca observa clases para dar retroalimentación (TALIS, 2013).

Una de las mesas de trabajo que se desarrollaba paralelamente a la discusión de la ley era la de agobio laboral. En Chile, un profesor con 44 horas cronológicas contratadas debe 
cumplir 33 frente a aula -43 horas pedagógicas-, y los recreos, reemplazos, reuniones, etc., cuentan como horas no lectivas, alcanzando el $25 \%$ del tiempo contratado, 11 horas. La ley incrementa a $30 \%$ las horas no lectivas, es decir, agrega 2 horas, y la obligación de destinar un $40 \%$ del tiempo a la preparación y evaluación de la enseñanza, y atención de estudiantes y padres, entre otras.

La posibilidad de llegar a un $40 \%$ de tiempo no lectivo se establece solo para profesores de estudiantes vulnerables de primer ciclo básico de establecimientos públicos, vía subvención escolar preferencial (SEP). Para los demás, eventuales incrementos quedan sujetos a indicadores económicos, y a partir del año 2020. Lejos aún del 50\%/50\% que caracteriza a los países de la OCDE, y engorrosa manera de no asegurar el cambio que se requiere, todo parece indicar la necesidad de buscar otra fórmula.

También, según datos de la OCDE, el año 2010, el tamaño promedio de la clase en los países miembros era de poco más de 20 , llegando en Chile a cerca de 30 , de los más altos de la organización, siendo superado sólo por Corea, Japón, Indonesia y China. Los datos de la prueba PISA indican que los sistemas que ponen foco en la calidad de los profesores y tienen cursos más pequeños tienden a obtener mejores resultados. Sin embargo, independientemente de esta evidencia, el impacto de una menor cantidad de estudiantes en sala para el manejo de grupo y la salud del maestro justifica por sí sola la adopción de una medida en tal sentido.

Respecto del número de estudiantes por sala, la ley no se pronuncia, pero es objeto de análisis en la mesa de trabajo correspondiente. Países con mejores indicadores educativos que los nuestros tienen cifras sustancialmente inferiores. Adicionalmente, se requiere discutir acerca del número de estudiantes totales atendidos por un profesor. En las mismas 40 horas un profesor puede tener la jefatura de un curso, y atender en la semana a 40 estudiantes, en los distintos subsectores, o bien puede hacer clases de un subsector en 8 cursos distintos, debiendo por ejemplo, corregir 320 pruebas de estudiantes distintos.

Finalmente, respecto de la necesidad de asegurar la distribución equitativa de profesores, una buena idea con una débil implementación: la ley incrementa los montos de asignaciones para quienes opten por desempeñarse en establecimientos con altos índices de vulnerabilidad, pero la cifra es muy poco significativa. Diversos estudios muestran la existencia de una mayor concentración de profesores con antecedentes de formación más precarios en escuelas más vulnerables, y con débiles resultados en mediciones estandarizadas, y de profesores con mejores antecedentes académicos en escuelas más aventajadas (Ruffinelli \& Guerrero, 2009; Meckes \& Bascopé, 2012). La ley ofrece escasas posibilidades de revertir esto y, respecto de las condiciones del ejercicio docente, hace particularmente pocos avances, generándose un discurso que no se condice con las condiciones ofrecidas y que tensiona el trato 'profesional'.

\subsubsection{Retiro de la carrera: un incómodo silencio}

La ley no alude al retiro. Sólo en la última etapa de discusión en la cámara baja se introdujo una indicación para la incorporación de un bono de incentivo permanente al retiro. El ejecutivo esgrimió que se trataba de un ardid político, de un costo imposible de asumir. La Comisión de Hacienda de la Cámara de Diputados eliminó el artículo. Queda en evidencia el profundo problema previsional de todos los trabajadores chilenos, que no es privativo de los maestros, y también de la necesidad de levantar argumentos más poderosos que los económicos para definir que no hace falta regular el retiro de los profesores chilenos. 


\section{SÍNTESIS Y ALGUNAS REFLEXIONES FINALES}

A partir de estas páginas, es posible identificar en la ley una inspiración en el enfoque profesional de tipo orgánico que se combina con elementos de la perspectiva gerencialista del desarrollo profesional, observándose algunas dimensiones que aparecen tensionadas o ambiguas, coexistiendo en ellas elementos de ambos tipos.

En el grupo de las políticas vinculadas a un desarrollo profesional orgánico se cuentan dimensiones claves: a) regulación del ingreso a carreras pedagógicas, orientada a revertir el enfoque mercantil en la selección de estudiantes; b) inducción a la iniciación, recuperando la mirada de construcción colaborativa, mediada y reflexiva de la profesión; c) progresión en la carrera, con un sistema de reconocimiento del desarrollo profesional que sintoniza con lógicas orgánicas, mejorando al inicio las remuneraciones y ligando su incremento posterior a evidencias de desarrollo profesional basado en perfeccionamiento gratuito y pertinente (es decir, no descansa únicamente en el incentivo, sino que ofrece apoyo); y d) formación continua como derecho, a partir de criterios de pertinencia y calidad, desde una perspectiva integral.

En el grupo de políticas con orientación gerencialista se identifican: a) regulación de la calidad de los egresados, que focaliza en las instituciones y la calidad de la oferta, pero enfatiza en los instrumentos de control: acreditación y evaluaciones a los estudiantes; y b) marginal mejoramiento de condiciones del ejercicio profesional, tensionando así el discurso del trato 'profesional'.

Finalmente, entre las dimensiones de la ley que resultan ambiguas se cuentan: a) regulación de la oferta formativa, orgánica al regular las condiciones para la calidad de esta oferta, pero manteniendo el enfoque mercantil en la no regulación de la cantidad de la oferta; b) ingreso automático a la profesión: orgánico al fortalecer la responsabilidad institucional por sobre la individual y apostar a la formación especializada de calidad por sobre la certificación, pero manteniendo la disposición que permite que cualquier profesional puede ejercer la docencia, situación propia de los enfoques gerencialistas; y c) evaluación docente, orgánica cuando amplía la evidencia que recoge el portafolio, pero mantiene la evaluación individual, debilitando la visión de profesionalización orgánica.

En síntesis, se trata de una ley de alto valor, marcadora de un hito histórico, que aborda la profesión docente de manera integral, orientándose a la profesionalización de la docencia desde una perspectiva que combina elementos de las perspectivas orgánica y gerencialista, pero fundamentalmente concebida desde el primer enfoque, con silencios y ambigüedades que tienden a debilitar su carácter orgánico, pero que en su conjunto y contexto se muestran ante todo, perfectibles y de evidente valor comparativo respecto de la situación anterior a su promulgación.

De ahora en más, apremia la mirada acuciosa sobre su implementación, interpretaciones, efectos y oportunidades de mejoramiento, la evaluación en terreno acerca de la capacidad de la ley para desarrollar la profesión docente que el país demanda.

\section{REFERENCIAS BIBLIOGRÁFICAS}

Abbott, A. (1988). The system of professions: an essay on the division of expert labor. Chicago: The University of Chicago Press. DOI:10.1017/S0007680500081034 
Estudios Pedagógicos XLII, N 4: 261-279, 2016

LEY DE DESARROLLO PROFESIONAL DOCENTE EN CHILE: DE LA PRECARIZACIÓN SISTEMÁTICA A LOS

LOGROS, AVANCES Y DESAFÍOS PENDIENTES PARA LA PROFESIONALIZACIÓN

Alexander, R. (2010). Children, their World, their Education, Oxon: Routledge. DOI: 10.1111/j.10990860.2010.00300.x

Alexander, R. (2011). Evidence, rhetoric and collateral damage: the problematic pursuit of 'world class' standards', Cambridge Journal of Education, vol. 41 (3), 265-286. DOI:10.1080/030576 4X.2011.607153

Arévalo; A.; Gysling, J. \& Reyes L. (2013). Bases para una propuesta de formación inicial docente de educación básica. Cuadernos de trabajo $\mathrm{N}^{\circ} 1$, Santiago: Departamento de Estudios Pedagógicos, Facultad de Filosofía y Humanidades, Universidad de Chile.

Ávalos, B. (2013). ¿Héroes o Villanos? La profesión docente en Chile. Santiago: Ed. Universitaria.

Bellei, C. (2001) El talón de Aquiles de la reforma educativa, en Martinic, S. y Pardo, M. (eds.), Economía política de las reformas educativas en América Latina, Santiago, PREAL-CIDE.

Bravo, D; Falck, D; González, R; Manzi, J. y Peirano, C. (2008). La relación entre la evaluación docente y el rendimiento de los alumnos: Evidencia para el caso de Chile. Recuperado el 24 de agosto de 2016, de http://www.ceppe.cl/images/stories/recursos/ publicaciones/Jorge\%20Manzi/La-relacin-entre-la-evaluacion-docente-y-el-rendimiento-delos-alumnos.pdf

Chile, (1997). Texto refundido, coordinado y sistematizado de la ley $\mathrm{n}^{\circ} 19.070$ que aprobó el estatuto de los profesionales de la educación, y de las leyes que la complementan y modifican. Recuperado el 23 de diciembre de 2015 desde http://bcn.cl/1lzfi

CIDE (2008). VII Encuesta CIDE a actores del sistema educativo: Informe final: 2008. Recuperado el 27 de noviembre de 2015 desde http://biblioteca.uahurtado.cl/cgi-bin/wxis.exe?IsisScript=/ ujah/cided/bdos. $x$ is \&base $=$ cided $\&$ rango $=1 \& \mathrm{mfn}=000248$

CIDE (2010). VIII Encuesta CIDE a actores del sistema educativo: Informe final: 2010. Recuperado el 27 de noviembre de 2015 desde http://biblioteca.uahurtado.cl/cgi-bin/wxis.exe?IsisScript=/ ujah/cided/bdos. xis \&base $=$ cided \&rango $=1 \& \mathrm{mfn}=000720$

Cox, C., Meckes, L. \& Bascopé, M. (2010). La institucionalidad formadora de profesores en Chile en la década del 2000: velocidad del mercado y parsimonia de las políticas. Pensamiento Educativo, vol. 46-47, 205-245.

Darling-Hammond, L. (2012). Educar con calidad y equidad: los dilemas del siglo XXI. Santiago: Fundación Chile.

Day, Ch.; Guy, Q. (2010). The New lives of teachers. London: Routledge.

Dubet, F., Bergounioux, A., Duru Bellat, M. (1999). Le collège de l'an 2000: rapport à la ministre déléguée chargée de l'enseignement scolaire. Paris, Documentation Française.

CPEIP, Ministerio de Educación. (2008). Marco para la buena enseñanza. Santiago: MINEDUC.

CPEIP, Ministerio de Educación. (2011). Estándares orientadores para egresados de pedagogía en educación básica. Santiago: MINEDUC.

CPEIP, Ministerio de Educación (2012). Estándares orientadores para egresados de pedagogía en educación media. Santiago: MINEDUC.

CHILE (2009). Ley General de Educación No 20.370 del 17 de agosto de 2009, artículo 46, letra 'g' que establece los criterios de idoneidad del personal docente y no docente en los establecimientos educativos. Biblioteca del Congreso Nacional de Chile. Recuperado el 18 de diciembre de 2015 desde http://www.leychile.cl/Navegar/?idNorma=1006043\&idVersion=2009-09-12\&idParte

Domínguez, M., Bascopé, M., Carrillo, C, Lorca, E., Olave, G., \& Pozo, M. (2012). Procesos de acreditación de pedagogías: un estudio del quehacer de las agencias. Calidad en la educación, vol.36, 53-85. DOI: $10.4067 /$ S0718-45652012000100002.

Feldfeber, M. (2007). La regulación de la formación y el trabajo docente: un análisis crítico de la "agenda educativa" en América Latina. \&EVBBítP e Sociedade, 28 (99), 444-465.

Gunter, H. (2008). Policy and workforce reform in England, Educational Management Administration \& Leadership, 2 (36), 253-270.

Hargreaves, A. \& Fullan, M. (2012). Professional capital: Transforming teaching in every school. 
New York, NY: Teachers College Press.

Ingersoll, R. (2011). Estudio comparado sobre la formación y antecedentes académicos de docentes en seis naciones. Serie Documentos, $\mathrm{N}^{\circ} 56$. Santiago: PREAL.

Lang, V. (2006). La construcción social de las identidades profesionales de los docentes en Francia: enfoques históricos y sociológicos. En: TENTI, E. (Comp.) El oficio de docente: vocación, trabajo y profesión en el siglo XXI. Buenos Aires, Siglo Veintiuno, 71-118.

Meckes, L. \& Domínguez, P. (2011). Análisis y propuestas para la acreditación de Pedagogías en Chile. Calidad en la Educación, vol.34, 165-183.

Meckes. L. \& Bascopé. M. (2012). Uneven Distribution of Novice Teachers in the Chilean Primary School System, Education Policy Analysis Archives, 20 (30), 1-27. DOI: http://dx.doi. org/10.14507/epaa.v20n30.2012

Munita, J. (2010) Elección de carrera y contexto social, mimeo.

OCDE (2004) Revisión de Políticas Nacionales de Educación: Chile. Paris: OCDE

OCDE (2009) Los Docentes importan. Atraer, formar y conservar a los docentes eficientes. Paris: OCDE.

OECD (2014), Talis 2013 results: An international perspective on teaching and learning, TALIS, OECD Publishing.

Osborne, D. \& Gaebler, T. (1993) Reinventing Government. How the Entrepreneurial Spirit is Transforming The Public Sector, Nueva York: Plume.

Ortúzar, M. S., Flores, C., Milesi, C., \& Cox, C. (2009). Aspectos de la formación inicial de docentes y su influencia en el rendimiento académico de los alumnos. En Camino al Bicentenario. Concursos Políticas Públicas para Chile 2009, (pp. 155-186). Santiago: Ediciones Universidad Católica.

Ortúzar, S., Flores, C., Milesi, C., Müller, M., \& Ayala, P. (2011). Diseño de un programa de inducción en Chile para profesores en sus primeros años de ejercicio docente. En Camino al Bicentenario. Concursos Políticas Públicas para Chile 2011. (pp. 267-305). Santiago: Ediciones Universidad Católica.

Ruffinelli, A. \& Guerrero A. (2009) Círculo de segmentación del sistema educativo chileno: destino laboral de egresados de pedagogía en Educación Básica, Calidad en la Educación, 31, 19-44.

Ruffinelli, A. (2013). La calidad de la formación inicial docente en Chile: la perspectiva de los profesores principiantes. Calidad en la educación, v.39, 118-154. DOI: 10.4067/S071845652013000200005

Ruffinelli, A. (2014). Dificultades de la iniciación docente ¿Iguales para todos? Estudios pedagógicos, vol. XI (1), 7-24. DOI: 10.4067/S0718-07052014000100014

Sisto, V. (2011). Nuevo profesionalismo y profesores: una reflexión a partir del análisis de las actuales políticas de 'profesionalización' para la educación en Chile. Signo y Pensamiento, 23 (59), 178-192.

Tenti Fanfani, E. (2006). Profesionalización docente: consideraciones sociológicas. En E. Tenti Fanfani (Ed.) El oficio de docente: Vocación, trabajo y profesión en el SXXI. (pp. 119-142). Buenos Aires: Siglo Veintiuno editores.

Valdebenito, M. (2013). IX Encuesta Cide: El diagnóstico a la justicia y equidad en la educación chilena. Mensaje, vol. 618, 48-49. 
